\title{
Information Theoretic Analysis of OFDM/OQAM with Utilized Intrinsic Interference
}

\author{
R. Razavi, Member, IEEE, P. Xiao, Senior Member, IEEE, R. Tafazolli, Senior Member, IEEE,
}

\begin{abstract}
In this paper, the capacity of OFDM/OQAM with isotropic orthogonal transfer algorithm (IOTA) pulse shaping is evaluated through information theoretic analysis. In the conventional OFDM systems the insertion of a cyclic prefix (CP) decreases the system's spectral efficiency. As an alternative to OFDM, filter bank based multicarrier systems adopt a proper pulse shaping with good time and frequency localisation properties to avoid interference and maintain orthogonality in real field among sub-carriers without the use of $\mathrm{CP}$. We evaluate the spectral efficiency of OFDM/OQAM systems with IOTA pulse shaping in comparison with conventional OFDM/QAM systems, and our analytical model is further extended in order to gain insights into the effect of utilizing the intrinsic interference on the performance of our system. Furthermore, the spectral efficiency of OFDM/OQAM systems is analyzed when the effect of intersymbol and inter-carrier interference is considered.
\end{abstract}

Index Terms-OFDM/OQAM, intrinsic interference.

\section{INTRODUCTION}

Orthogonal frequency division multiplexing (OFDM) is a multicarrier technique [1], [2] employed in several wireless standards due to its robustness in combatting multipath fading channels. By dividing a wideband fading channel into a parallel of flat narrowband channels, OFDM is able to mitigate the harmful effects of multipath fading. However, this property is achieved by extending the length of the OFDM symbol and introducing a CP. This part of the OFDM symbol contains redundant information and results in a reduced spectral efficiency and increased power consumption. An alternative is through the use of pulse shaping filters. With offset modulations, for example, offset quadrature amplitude modulation (OQAM), the orthogonality can be maintained with proper pulse shaping design. These systems are commonly referred to as OFDM/OQAM systems [3], [4], [5].

We need to choose pulses with good time and frequency localisation (TFL) properties. The localisation in time aims to limit inter-symbol interference (ISI) and the localisation in frequency aims to limit inter-carrier interference (ICI) caused by, e.g., Doppler effects and carrier frequency offset. Among different prototype filters, such as half-cosine, root-raisedcosine [6], isotropic orthogonal transform algorithm (IOTA) [5], [7], extended Gaussian function (EGF) [8], etc., the localisation property is optimal with the IOTA function which has the same shape in time and frequency domains. Having a certain level of ISI (due to channel dispersion) and ICI when the channel is time varying is a common problem in multicarrier systems, however it is shown that OFDM/OQAM

The authors are with the Institute for Communication Systems, University of Surrey, GU2 7XH, UK (e-mail: [R.Razavi, P.Xiao, R.Tafazolli] @surrey.ac.uk). with IOTA pulse shaping is more resistant to these interferences [9]. OFDM/OQAM systems provide orthogonality in the real domain, therefore, instead of using complex baseband symbols as in OFDM/QAM scheme, real valued symbols modulated by offset QAM are transmitted on each sub-carrier [5]. OFDM/OQAM systems and conventional OFDM systems in a realistic mobile communication context (in both time and frequency dispersive channels) have been compared in [10], [11], [12] where it was shown that the performance advantage of OFDM/OQAM with IOTA pulse shaping over conventional OFDM can be up to $4.5 \mathrm{~dB}$ depending on different types of wireless propagation channels.

Despite all the advantages, the currently developed OFDM/OQAM system is far from its best achievable performance. There are still gains to be attained if some of its special properties can be leveraged to maximum advantage. One of these properties is its intrinsic interference which has not yet been utilized in detecting the desired symbols. This motivates us to investigate the system's capacity limits, to provide us with guidelines for the design of coding, channel estimation and demodulation techniques for OFDM/OQAM systems.

In practical systems an adaptive coding and modulation (ACM) technique for OFDM systems is used [13]. With ACM it is possible to dynamically modify the coding rate and modulation scheme for each frame, according to the measured channel conditions where the frame is received. This technique provides better channel protection and exploits the capacity of broadband frequency selective channels. The ACM technique can be applied to OFDM/OQAM systems in a similar manner.

In this contribution we investigate the information-theoretic capacity of OFDM/OQAM systems with IOTA pulse shaping and compare it with that of OFDM/QAM systems. The capacity is calculated for input with Gaussian distribution which is never the case in practice. We thus take into account realistic assumptions to improve the accuracy of the proposed analysis. Furthermore, the derived model is later extended by incorporating intrinsic interference utilization and the effect of ISI and ICI is analyzed.

The rest of the paper is outlined as follows. In Sec. II after presenting the system model for an OFDM/OQAM system, we derive its information-theoretic capacity. The extended model where the intrinsic interference is utilized is presented in Sec. III. In Sec. IV we derive the capacity of an OFDM/OQAM system with IOTA pulse shaping when the system suffers from ISI and ICI. Finally, conclusions are drawn in Sec. V. 


\section{CAPACITY OF IOTA SYSTEM}

We assume a multicarrier system with $N$ subcarriers. The transmitted signal for an OFDM/OQAM system will be

$$
s(t)=\sum_{n \in \mathbb{Z}} \sum_{m=0}^{N-1} a_{n, m} g_{n, m}(t)
$$

where $a_{m, n}$ is the symbol modulated by the $m$-th sub-carrier at the symbol time of index $n$, and

$$
\begin{aligned}
g_{n, m}(t) & =g\left(t-n \tau_{0}\right) \\
& \left.\exp \left(j[\pi(m+n) / 2)+2 \pi m \nu_{0} t\right]\right), \nu_{0} \tau_{0}=1 / 2,
\end{aligned}
$$

representing the synthesis basis, which is obtained by the time frequency translation of the IOTA prototype function $g(t)$. In an OFDM system, the synthesis basis can be expressed as

$$
g_{n, m}(t)=g\left(t-n\left(T+T_{c p}\right)\right) \exp (j 2 \pi m F t), \quad T F=1
$$

where $T, T_{c p}$ and $F$ are the symbol duration, the cyclic prefix duration and inter-carrier frequency spacing, respectively, and $g(t)$ is the rectangular function. After passing through the doubly dispersive channel, the received signal will be

$$
r(t)=\sum_{n, m} a_{n, m} \iint H(\tau, \nu) g_{n, m}(t-\tau) e^{j 2 \pi \nu t} d \nu d \tau+v(t),
$$

where $H(\tau, \nu)$ is the Fourier transform of channel impulse response. The integration intervals depend on the channel delay spread, $\tau_{\max }$, and the maximum Doppler frequency $f_{D}$. Without loss of generality, we assume $a_{l, k}$ is the symbol to be detected. The demodulator output can be expressed as [14]

$$
a_{l, k}=\Re\left\{\left\langle r(t), g_{l, k}(t)\right\rangle\right\} .
$$

The IOTA demodulator can be implemented by filter banks followed by an fast Fourier transform (FFT) block, as shown in Fig. 1. The system uses offset modulation for which the $I$ and $Q$ signal components are mis-aligned by half a symbol interval. Like in the transmitter, the FFT operations are conducted separately for the $I$ and $Q$ components of OQAM symbols. This block diagram of OFDM/OQAM systems shows that the complexity of an OFDM/OQAM system is roughly twice compared to that of the conventional OFDM while having various advantages as mentioned earlier. As indicated by this figure, the imaginary part of the equalised and phase corrected signal from the $I$ channel and the real part of the equalised and phase corrected signal from the $Q$ channel are regarded as intrinsic interference and not used for data detection. In order to find the theoretical gain achieved by OFDM/OQAM systems with IOTA pulse shaping compared to OFDM/QAM we shall now derive the capacity of an OFDM/OQAM system. According to [7] the tolerance of OQAM modulation to delay is approximately equal to $86 \%$, therefore we can have ISI free communication as long as $\tau_{\max } \leqslant 14 \% T$. Under this condition, considering that OFDM/OQAM systems avoid interference and maintain orthogonality in real field among sub-carriers, the capacity of an OFDM/OQAM system can be calculated by using the capacity formula for parallel channels

$$
C=\frac{1}{T} \sum_{k \in \mathbb{K}} \log _{2}\left(1+\mathrm{SNR}_{k}\right)
$$

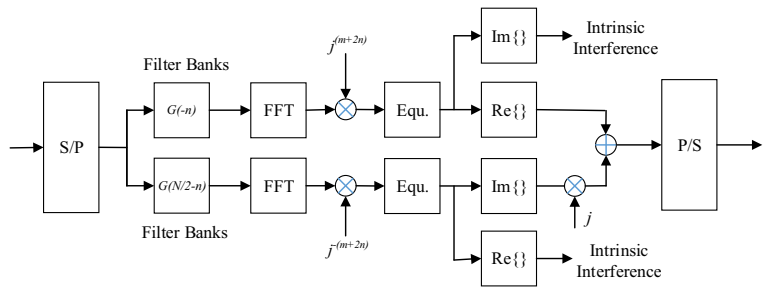

Fig. 1. Block diagram of the IOTA receiver.

where $T$ is the symbol duration and $\mathrm{SNR}_{k}$ is the signal to noise ratio (SNR) of subchannel $k$. $\mathbb{K}$ is the set of subcarrier indices for those used subcarriers, i.e., $\mathbb{K} \subseteq\{0,1, \ldots, N-1\}$. In the case when each subchannel experiences flat fading, let $h_{k}(i)$ represent the complex channel gain of fading process at subcarrier $k$, the ergodic capacity under fading channel is

$$
C=\mathbb{E}\left[\frac{1}{T} \sum_{k \in \mathbb{K}} \log _{2}\left(1+\frac{P_{S}^{k}}{N_{0}^{k}}\left|h_{k}\right|^{2}\right)\right],
$$

where $P_{S}^{k}$ and $N_{0}^{k}$ are the power of the received symbol and noise variance for subcarrier $k$, respectively. In order to have a more practical evaluation we take into account finite size constellations.

The SNR gap approximation, first introduced by D. Forney, is a convenient way of expressing the achievable rates when a suboptimal transmission scheme is used that aims at a given probability of symbol error, $P_{e}$ [15]. Thus, using the gap approximation the rate achieved by a discrete constellation over an additive white Gaussian noise (AWGN) channel can be expressed in a form similar to Shannon's capacity formula. In this paper, to study the effect of modulation on the capacity of OFDM/OQAM systems we adopt the SNR gap concept. To achieve a given data rate at a given probability of error with a specific modulation scheme, a value of SNR higher than that suggested by Shannon's capacity is required. This extra amount of power is called the SNR gap, denoted in this paper by $\Gamma_{\text {mod }}$. The SNR gap is widely studied in the single user case for different modulation schemes by considering the error probability relation with the modulation order and SNR. Please notice that by separating real and imaginary components in offset modulations the overall data rate won't change comparing to the conventional modulation for OFDM systems. Therefore, the SNR gap concept will be valid for OFDM/OQAM systems as well. The maximum achievable rate for an OFDM/OQAM system with finite size constellation modulation under AWGN channel can be expressed as follows

$$
\begin{aligned}
R & =\frac{1}{T} \sum_{k \in \mathbb{K}} \log _{2}\left(1+\frac{\mathrm{SNR}_{k}}{\Gamma_{\bmod }}\right) \\
& =\frac{1}{T} \sum_{k \in \mathbb{K}} \log _{2}\left(1+\frac{P_{S}^{k}}{\Gamma_{\bmod } N_{0}^{k}}\right),
\end{aligned}
$$

For a system with $M$-QAM modulation, $\Gamma_{m o d}$ is calculated as follows [16]:

$$
\Gamma_{\text {mod }}\left(P_{e}, M\right)=\left[Q^{-1}\left(\frac{M P_{e}}{2(M-1)}\right)\right]^{2} / 3 .
$$


In Fig. 2, the spectral efficiency of an OFDM/OQAM system is compared with that of an OFDM/QAM system based on IEEE 802.11a specifications. The FFT size of both systems is equal to 64. The modulation scheme is 16-QAM for the OFDM system and 16-OQAM for the IOTA system. The OFDM symbol duration is equal to $4 \mu s$ and the CP duration is determined using channel delay spread. The results show that the OFDM/OQAM system benefits from a gain of 1.1 $d B$ at spectral efficiency of 2 bits $/ \mathrm{s} / \mathrm{Hz}$ compared with the OFDM/QAM system.

\section{CAPACITY OF OFDM/OQAM SYSTEMS WITH UTILIZED INTRINSIC INTERFERENCE}

As mentioned earlier in the IOTA receiver the $Q$ component of the first equalizer output and the $I$ component of the second second equalizer are called intrinsic interference and usually discarded in the modulation process. In [17], [18] the intrinsic interference is exploited to improve the channel estimation. The authors presented a general theoretical framework for interference approximation method (IAM) preamble design and applied it to identify the optimal IAM preamble sequence which resulted in a higher gain. In [19], the intrinsic interference is exploited instead of being discarded at the receiver side in order to improve the equalization performance. It was shown in the paper that the real and the time-shifted imaginary components can be highly correlated. This analysis led to a new simple and efficient equalizer utilizing this correlation. Also in [20] an analysis on intrinsic interference of a causal multirate OFDM/OQAM systems is conducted. These findings reveal the fact that the intrinsic information can be exploited for performance improvements.

In this section we will analyze the effect of complete utilization of intrinsic interference on the capacity of OFDM/OQAM systems. The additional information extracted from the intrinsic interference would increase the maximum achievable rate, due to the added power of the desired signal, i.e.,

$$
C=\frac{1}{T} \sum_{k \in \mathbb{K}} \log _{2}\left(1+\frac{P_{S}^{k}+P_{I}^{k}}{\Gamma_{\text {mod }} N_{0}^{k}}\right),
$$

where $P_{I}^{k}$ is intrinsic interference's power at subcarrier $k$.

The intrinsic interference corresponding to the detected symbol $a_{l, k}$ can be expressed as [21]

$$
\begin{aligned}
I_{l, k} & =\sum_{(n, m) \neq(l, k)} a_{n, m} \\
& \iiint_{\mathbb{R}} H(\tau, \nu) g_{n, m}(t-\tau) e^{j 2 \pi \nu t} g_{n, m}^{*}(t) d \nu d \tau d t .
\end{aligned}
$$

By applying auto-ambiguity function

$$
A_{g}(\tau, \nu)=\int_{\mathbb{R}} e^{-j 2 \pi \nu t} g(t+\tau / 2) g^{*}(t-\tau / 2) d t,
$$

the interference can be reformed as

$$
\begin{aligned}
& I_{l, k}=\sum_{(p, q) \neq(0,0)} a_{l+p, k+q} \iint_{\mathbb{R}} H(\tau, \nu) A_{g}^{*}\left(q \tau_{0}+\tau, p \nu_{0}+\nu\right) \\
& e^{j \pi\left(q \tau_{0} \nu-p \nu_{0} \tau\right)} e^{j \pi\left(2 k \tau_{0} \nu-2 l \nu_{0} \tau+\tau \nu\right)} d \nu d \tau,
\end{aligned}
$$

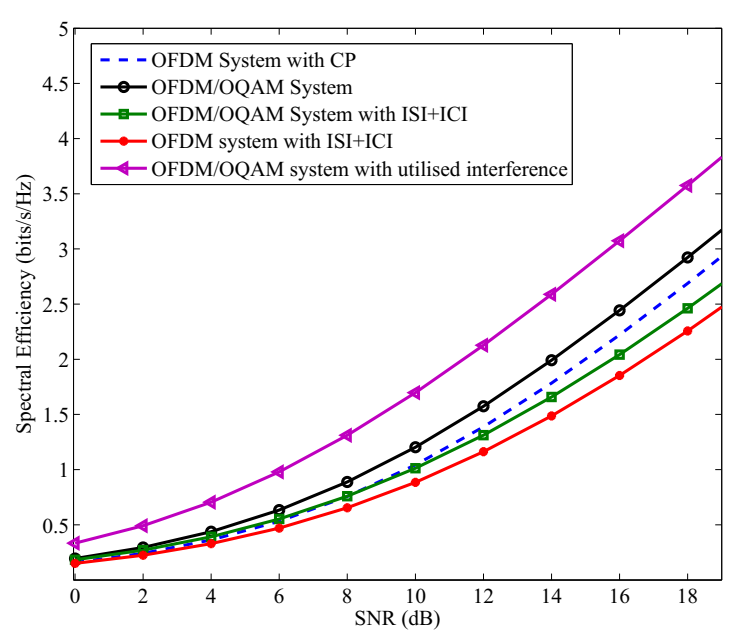

Fig. 2. Spectral efficiency of an OFDM/OQAM system.

where $p=n-l$ and $q=m-k$. Assuming that the channel is moderately dispersive $\left(\tau_{\max } \ll \tau_{0}\right.$ and $\left.f_{D} \ll \nu_{0}\right)$, the ambiguity function has relatively low variation around the lattice points $\left(q \tau_{0}, p \nu_{0}\right)$. Thus, for $|\tau| \leqslant \tau_{\max },|\nu| \leqslant f_{D}$ we have

$$
\begin{aligned}
& I_{l, k}=\sum_{(p, q) \neq(0,0)} a_{l+p, k+q} A_{g}^{*}\left(q \tau_{0}, p \nu_{0}\right) \\
& \quad \iint_{\mathbb{R}} H(\tau, \nu) e^{j \pi\left(2 k \tau_{0} \nu-2 l \nu_{0} \tau+\tau \nu\right)} d \nu d \tau .
\end{aligned}
$$

Assume all the transmitted symbols are independent with uniform energy, i.e.,

$$
E\left\{a_{n, m} a_{n^{\prime}, m^{\prime}}^{*}\right\}= \begin{cases}1, & n=n^{\prime} \text { and } m=m^{\prime} \\ 0, & \text { otherwise. }\end{cases}
$$

The energy of the intrinsic interference at subcarrier $k$ will be

$$
\begin{aligned}
& E_{I}^{k}=E\left\{I_{l, k} I_{l, k}^{*}\right\} \\
& =E\left\{\sum_{(p, q) \neq(0,0)}\left|A_{g}\left(q \tau_{0}, p \nu_{0}\right)\right|^{2} \iiint \int_{\mathbb{R}} H(\tau, \nu) H^{*}\left(\tau^{\prime}, \nu^{\prime}\right)\right. \\
& \left.e^{j \pi\left(2 k \tau_{0} \nu-2 l \nu_{0} \tau+\tau \nu-2 k \tau_{0} \nu^{\prime}+2 l \nu_{0} \tau^{\prime}-\tau^{\prime} \nu^{\prime}\right)} d \nu d \tau d \nu^{\prime} d \tau^{\prime}\right\} \\
& =\sum_{(p, q) \neq(0,0)}\left|A_{g}\left(q \tau_{0}, p \nu_{0}\right)\right|^{2} \iiint \int_{\mathbb{R}} E\left\{H(\tau, \nu) H^{*}\left(\tau^{\prime}, \nu^{\prime}\right)\right\} \\
& \left.e^{j \pi\left(2 k \tau_{0} \nu-2 l \nu_{0} \tau+\tau \nu-2 k \tau_{0} \nu^{\prime}+2 l \nu_{0} \tau^{\prime}-\tau^{\prime} \nu^{\prime}\right)} d \nu d \tau d \nu^{\prime} d \tau^{\prime}\right\} \\
& =\sum_{(p, q) \neq(0,0)}\left|A_{g}\left(q \tau_{0}, p \nu_{0}\right)\right|^{2} \iint_{\mathbb{R}} S_{h}(\tau, \nu) d \nu d \tau,
\end{aligned}
$$

where for a wide sense stationary uncorrelated scattering (WSSUS) channel, $S_{h}(\tau, \nu)$ is defined as the scattering function [22]. One of the most commonly used WSSUS doubly dispersive channels, in which an exponential delay power profile and a U-shaped Doppler power spectrum are assumed, is defined by its scattering function in the following way [22]

$$
S_{h}(\tau, \nu)=\frac{e^{\frac{-|\tau|}{\tau_{R M S}}}}{\tau_{R M S}} \frac{1}{\pi f_{D} \sqrt{1-\left(\frac{\nu}{f_{D}}\right)^{2}}},
$$


where $\tau_{R M S}$ is the RMS delay spread. The power of intrinsic interference at subcarrier $k, P_{I}^{k}$, can be found by dividing the calculated energy in (16) by symbol duration $T$.

The analytical results are shown in Fig. 2 where one can see how utilizing the intrinsic interference can improve the spectral efficiency of the system. An SNR gain of $2.9 \mathrm{~dB}$ is achieved at spectral efficiency equal to $2 \mathrm{bits} / \mathrm{s} / \mathrm{Hz}$ compared to the OFDM/OQAM without interference utilization. This result motivated further studies on methods that can extract the information from the intrinsic interference of an OFDM/OQAM system to improve its performance. [23]

\section{OFDM/OQAM UNDER FREQUENCY SELECTIVE FADING CHANNELS}

In previous sections we have analyzed a fading model which is appropriate if the Nyquist sampling period is large compared to delay spread of multi-paths in the received signal, so that individual paths are not resolvable in the sampled system. For wideband applications, the multi-paths can be resolved, and hence channel has memory. The appropriate model in this case is the frequency selective fading channel. In this section, we extend our previous results for OFDM/OQAM to this model.

We denote $h[l]$ as the $l^{\text {th }}$ channel tap. In order to consider the ISI and ICI effect we follow the procedure provided in [24]. The discrete-time demodulated signal at $\left(n_{0}, m_{0}\right)$ can be expressed as [25]

$$
y_{n_{0}, m_{0}}=\beta_{m_{0}} a_{n_{0}, m_{0}}+J_{n_{0}, m_{0}}+v_{n_{0}, m_{0}}
$$

with

$$
\beta_{m_{0}}=\sum_{l} h[l] e^{-j \frac{2 \pi}{N} m_{0} l} A_{g}[-l, 0],
$$

where $A_{g}[l, k]$ is the sampled version of the auto-ambiguity function $A_{g}(\tau, \nu)$. The complex-valued term $J_{n_{0}, m_{0}}$ which stands for the ISI (due to random propagation delay and multipath fading)+ICI (due to Doppler-spread of the channel) is

$$
\begin{aligned}
J_{n_{0}, m_{0}}= & \sum_{(p, q) \neq(0,0)} a_{n_{0}+p, m_{0}+q} e^{j\left[\frac{\pi}{2}(p+q+p q)+\pi q n_{0}\right]} \\
& \underbrace{\sum_{l} h[l] e^{-j \frac{\pi}{N}\left(2 m_{0}+q\right) l} A_{g}\left[-p \frac{N}{2}-l, q\right]}_{h_{p, q}\left(m_{0}\right)} .
\end{aligned}
$$

Therefore the power of ISI+ICI at the output of one-tap ZF equalizer can be derived according to [24] as

$$
\begin{aligned}
& P_{I S I+I C I}\left(m_{0}\right)= \\
& 2 \sigma_{b}^{2} \sum_{(p, q) \neq(0,0)}\left|\Re\left\{\frac{e^{j \frac{\pi}{2}(p+q+p q)} h_{p, q}\left(m_{0}\right)}{h_{m_{0}}}\right\}\right|^{2} .
\end{aligned}
$$

The choice of $(p, q)$ depends on the prototype filter. Considering that IOTA is optimized for time and frequency localization, the energy of its ambiguity function will not spread to a large neighborhood zone. The capacity for an IOTA system with ICI+ISI is

$$
C=\mathbb{E}\left[\frac{1}{T} \sum_{k \in \mathbb{K}} \log _{2}\left(1+\frac{P_{S}^{k}}{P_{I S I+I C I}^{k}+N_{0}^{k}}\left|h_{k}\right|^{2}\right)\right]
$$

Fig. 2 shows the effect of ISI+ICI on spectral efficiency of the OFDM/OQAM system when a channel model of SUI3 (Stanford University Interim) is applied. The CP duration $(0.8 \mu s)$ for OFDM symbol is less than the expected delay spread of the SUI-3 channel, therefore the OFDM system suffers from ISI+ICI. Also for the OFDM/OQAM we have $\tau_{\max } \geq 0.14 * 4(\mu s)$, therefore the OFDM/OQAM system will have the same level of average ISI+ICI as in the OFDM system. As it is shown for higher SNRs the spectral efficiency of conventional OFDM is lower than the one for OFDM/OQAM systems when both systems have ISI+ICI.

\section{Conclusions}

OFDM/OQAM systems can be designed to improve the power and spectral efficiencies of a wireless communication system. In this paper we carried out an informationtheoretic analysis of OFDM/OQAM systems with IOTA pulse shaping to gain insights into the gains that can be achieved by exploiting special properties inherent in OFDM/OQAM systems. First, we analyzed the capacity of an OFDM/OQAM system for inputs with Gaussian distribution. For practical considerations, we extended the formula to the case with finite size constellations. The comparison of spectral efficiency of an OFDM/OQAM system with an OFDM/QAM system shows that a gain of $1.1 \mathrm{~dB}$ at spectral efficiency of $2 \mathrm{bits} / \mathrm{s} / \mathrm{Hz}$ can be achieved. Furthermore, the effect of ISI+ICI on the spectral efficiency of OFDM/OQAM systems were evaluated and we have shown that in terms of spectral efficiency the OFDM/OQAM remained superior to the OFDM.

Our analytical model is further developed to analyze the effect of utilized intrinsic interference on the spectral efficiency of an OFDM/OQAM system, and is shown that compared to conventional OFDM systems a considerable gain of $4 d B$ in total at spectral efficiency of 2 bits $/ \mathrm{s} / \mathrm{Hz}$ can be achieved under SUI-3 channel. Those promising results motivate further research on the utilization of the intrinsic interference in order to explore the potential to attain the maximum performance gain in future OFDM/OQAM systems. [23]

\section{ACKNOWLEDGMENT}

This work was sponsored by the UK Engineering and Physical Sciences Research Council (EPSRC) under grant number EP/J017655/1. The authors would like to acknowledge its financial support.

\section{REFERENCES}

[1] B. Saltzberg, "Performance of an efficient parallel data transmission system," IEEE Trans. Commun. Technol., vol. 15, no. 6, pp. 805-811, 1967.

[2] J. A. C. Bingham, "Multicarrier modulation for data transmission: an idea whose time has come," IEEE Commun. Mag., vol. 28, no. 5, pp. 514, 1990.

[3] B. Hirosaki, "An orthogonally multiplexed QAM system using the discrete Fourier transform," IEEE Trans. Commun., vol. 29, no. 7, pp. 982-989, 1981.

[4] H. Bolcskei, P. Duhamel, and R. Hleiss, "Design of pulse shaping OFDM/OQAM systems for high data-rate transmission over wireless channels," in Proc. IEEE Int. Conf. Communications ICC '99, vol. 1, pp. 559-564, 1999. 
[5] B. Le Floch, M. Alard, and C. Berrou, "Coded orthogonal frequency division multiplex," Proc. IEEE, vol. 83, no. 6, pp. 982-996, 1995.

[6] N. J. Baas and D. P. Taylor, "Pulse shaping for wireless communication over time- or frequency-selective channels," IEEE Trans. Commun., vol. 52, no. 9, pp. 1477-1479, 2004.

[7] M. Alard, "Construction of a multicarrier signal," WO 96/35278, 1996.

[8] P. Siohan and C. Roche, "Cosine-modulated filterbanks based on extended Gaussian functions," IEEE Trans. Signal Process., vol. 48, no. 11, pp. 3052-3061, 2000

[9] B. Farhang, "OFDM versus filter bank multicarrier," IEEE Signal Process. Mag., vol. 28, no. 3, pp. 92-112, 2011.

[10] D. Lacroix, N. Goudard, and M. Alard, "OFDM with guard interval versus OFDM/offsetQAM for high data rate UMTS downlink transmission," in Proc. IEEE 54th Vehicular Technology Conf. (VTC 2001 Fall)., vol. 4, pp. 2682-2686, 2001.

[11] J. Du and S. Signell, "Comparison of CP-OFDM and OFDM/OQAM in doubly dispersive channels," in Proc. Future Generation Communication and Networking (FGCN 2007), vol. 2, pp. 207-211, 2007.

[12] K. Kongara, P. Smith, and S. Mann, "A comparison of CP-OFDM with IOTA-OFDM under typical system imperfections," in IET Seminar on Wideband and Ultrawideband Systems and Technologies: Evaluating current Research and Development, pp. 1-5, 2008.

[13] S. Stiglmayr, M. Bossert, and E. Costa, "Adaptive coding and modulation in an OFDM-TDMA communication system," in Vehicular Technology Conference, 1998. VTC 98. 48th IEEE, vol. 2, pp. 773-776, 1998

[14] P. Siohan, C. Siclet, and N. Lacaille, "Analysis and design of OFDM/OQAM systems based on filterbank theory," IEEE Trans. Signal Process., vol. 50, no. 5, pp. 1170-1183, 2002.

[15] J. Cioffi, Course notes for Digital Communication. Signal Processing. Standford Bookstore Custom Publishing, 2007.

[16] G. D. Forney, Jr. and G. Ungerboeck, "Modulation and coding for linear Gaussian channels," IEEE Trans. Inf. Theory, vol. 44, no. 6, pp. 2384 2415, 1998.

[17] J. Du and S. Signell, "Novel preamble-based channel estimation for OFDM/OQAM systems," in Proc. IEEE International Conference on Communications, 2009. ICC '09., pp. 1-6, 2009.

[18] J. Du, Pulse Shape Adaptation and Channel Estimation in Generalised Frequency Division Multiplexing Systems. PhD thesis, KTH, 2008.

[19] G. Ndo, H. Lin, and P. Siohan, "FBMC/OQAM equalization: Exploiting the imaginary interference," in Proc. IEEE 23rd Int Personal Indoor and Mobile Radio Communications (PIMRC) Symp, pp. 2359-2364, 2012.

[20] M. Sriyananda and N. Rajatheva, "Analysis of self interference in a basic FBMC system,” in Vehicular Technology Conference (VTC Fall), 2013 IEEE 78th, pp. 1-5, 2013.

[21] C. Lélé, J.-P. Javaudin, R. Legouable, A. Skrzypczak, and P. Siohan, "Channel estimation methods for preamble-based OFDM/OQAM modulations," Trans. on Emerging Telecommun. Tech., vol. 19, no. 7, pp. 741$750,2008$.

[22] J. Proakis, Digital Communications. McGram-Hill, 2000.

[23] "Intrinsic interference utilisation for FBMC-IOTA systems." International patent to be filed by $5 \mathrm{G}$ innovation centre, University of Surrey.

[24] H. Lin and P. Siohan, "Capacity analysis for indoor PLC using different multi-carrier modulation schemes," IEEE Trans. Power Del., vol. 25, no. 3, pp. 113-124, 2010.

[25] H. Lin, C. Lele, and P. Siohan, "Equalization with interference cancelation for hermitian symmetric OFDM/OQAM systems," in IEEE International Symposium on Powerline communications and its applications., 2008. 